\title{
Control of anesthesia based on singularly perturbed model
}

\author{
Sophie Tarbouriech, Isabelle Queinnec, Germain Garcia, Michel Mazerolles
}

\begin{abstract}
This paper deals with the control of anesthesia taking into account the positivity together with the upper limitation constraints of the variables and the target interval tolerated for the depth of anesthesia during a surgery. Due to the presence of multiple time scale dynamics in the anesthesia model, the system is re-expressed through a singularly perturbed system allowing to decouple the fast dynamics from the slow ones. Differently from general approaches for singularly perturbed systems, the control objective is then to control and accelerate the fast system without interest in modifying the slow dynamics. Thus, a structured state feedback control is proposed through quasi-LMI (linear matrix inequalities) conditions. The characterization of domains of stability and invariance for the system is provided. Associated convex optimization issues are then discussed. Finally, the theoretical conditions are evaluated on a simulated patient case.
\end{abstract}

\section{Introduction}

The principle of general anesthesia and drug delivery control during surgery corresponds to the suspension of consciousness (hypnosis), pain (analgesia) and movement (immobility). Indeed, to address these three main actions, a combination of drugs is used. In this paper we focus on the hypnosis problem only, with Propofol used as hypnotic drug. Even if this is an old problem (notion of closed-loop control appeared in the fifties), it remains largely open. Actually, medical practices remain yet in open loop and several researchers from the control community have been con-

Sophie Tarbouriech, Isabelle Queinnec, Germain Garcia

LAAS-CNRS, Université de Toulouse, CNRS, Toulouse, France e-mail: sophie.tarbouriech,isabelle.queinnec,germain.garcia@laas.fr

Michel Mazerolles

Département d'anesthésie-réanimation, CHU Toulouse, 31059 cedex, France e-mail: mazerolles.m@chu-toulouse.fr 
cerned with such applications and suggested advanced control techniques to move from open-loop control by the anesthesiologist to closed-loop control [13]. Hence, the control of the anesthetic state of a patient consists in adjusting the perfusion of hypnotics based on clinical indicators such as heart rate, blood pressure and BIS (Bispectral index). The control of anesthetic drugs injection for maintaining an adequate anesthetic state during surgery has been studied through several approaches. Among them, one can, for example, cite the use of PID controllers [17], adaptive control [11], model predictive control [9], LPV modeling and control [2], bifurcation analysis [20] and set-theoretic tools [7].

As for many biological systems, the design of an adequate control law should take into account some physical aspects such as patient variability, positivity constraints, output measurement availability, the presence of multiple time scales in the dynamics... Indeed, the dynamics of the drug evolution in the patient's body is usually described by a pharmacokinetic positive model with multiple time scales. In this paper, we use to represent this difference the framework of singularly perturbed systems [12]. Hence, the compartmental system describing the anesthesia model is re-expressed through a singularly perturbed system allowing to decouple the fast dynamics (blood, effect site) from the slow ones (muscles, fat). Differently from general approaches for singularly perturbed systems, the control objective is then to control and accelerate the fast system without interest in modifying the slow dynamics. Furthermore, the control design has to take into account the positivity together with the upper limitation constraints of the variables during a surgery. Thus, based on the results in [8] and [14], a structured state feedback control is proposed through theoretical matrix inequalities, which constitutes the main contribution of the paper. The characterization of domains of stability and invariance for the system is provided by using some relaxation schemes in order to obtain linear matrix inequalities (LMI) conditions. Associated convex optimization issues are then discussed.

The paper is organized as follows. Section 2.1 presents the compartment-based model, for which the presence of multiple time scale dynamics is pointed out. Then, the system is re-expressed through a singularly perturbed system allowing to decouple the fast dynamics from the slow ones. The general problem formulation is summarized in Section 2.2 and the theoretical conditions allowing to design the structured state feedback controller are provided in Section 3.1. Associated algorithms are then proposed in Section 3.2 in order to exhibit numerical solutions. Section 4 presents the patient case considered in order to illustrate the effectiveness, the drawback and the trade-off of the proposed solution. Finally, some concluding remarks in Section 5 end the paper.

Notation. For a matrix $P$ in $\mathbb{R}^{n \times n}$, the notation $P>0(P \geq 0)$ means that $P$ is symmetric positive (semi) definite. For a vector $x \in \mathbb{R}^{n}$, the notation $x \geq 0$ means that all the components of the vector are nonnegative. The superscript ' $T$ ' 'stands for matrix transposition, and the notation $\mathrm{He}(P)$ stands for $P+P^{T}$. The symbols $I$ and 0 represent the identity and the zero matrices of appropriate dimensions. 


\section{Patient model and problem formulation}

\subsection{Patient model}

It is well accepted that the model used to describe the evolution of drugs in a patient's body is a Pharmacokinetic/Pharmacodynamic (PK/PD) model, which is based on a three-compartment model [6]. Such a PK/PD model describes the distribution of the drugs between three compartments (blood, muscles and fat). Furthermore, the effect of drugs on the patient is expressed throughout the effect site, which represents the action of drugs on the brain and is related to the concentration in the central compartment through a first order dynamics [10], [2].

Hence, the compartmental model representing the circulation of the drug in the body can be written as follows ${ }^{1}$ :

$$
\dot{x}_{a n}=A_{0} x_{a n}+B_{0} u_{a n}
$$

with

$$
A_{0}=\left[\begin{array}{cccc}
-\left(a_{10}+a_{12}+a_{13}\right) & a_{21} & a_{31} & 0 \\
a_{12} & -a_{21} & 0 & 0 \\
a_{13} & 0 & -a_{31} & 0 \\
a_{e 0} / V_{1} & 0 & 0 & -a_{e 0}
\end{array}\right] ; B_{0}=\left[\begin{array}{l}
1 \\
0 \\
0 \\
0
\end{array}\right]
$$

In the vector $x_{a n}=\left[\begin{array}{llll}x_{1} & x_{2} & x_{3} & x_{4}\end{array}\right]^{\prime}, x_{1}, x_{2}, x_{3}$ are the masses in grams of the drug in the different compartments (blood, fat, muscle), $x_{4}$ is the effect site concentration and $u_{a n}$ is the infusion rate in $\mathrm{g} / \mathrm{min}$ of the anesthetic. The parameters $a_{i j} \geq 0, \forall i \neq j, i, j=1,2,3$, are the transfer rates of the drug between compartments. The parameter $a_{10}$ represents the rate of elimination from the central compartment. These parameters are functions of the patient characteristics (weight, age, height, ...). Several empirical models give the relation between those parameters and patient's characteristics [5]. One can cite, for example, the models of [15] or [16] related to Propofol (hypnotic drug) and Remifentanil (analgesic drug), respectively, to define a typical patient and to build uncertain models to represent the inter-patient variability.

Moreover, the depth of anesthesia indicator widely used by clinicians is the $B I S$ (the bispectral index), which is a signal derived from the EEG analysis. BIS quantifies the level of consciousness of a patient from 0 (no cerebral activity) to around 100 (fully awake patient). The relationship between the concentration at the effect site $x_{4}$ and the BIS can be described empirically by a decreasing sigmoid function [1]:

$$
B I S\left(x_{4}\right)=B I S_{0}\left(1-\frac{x_{4}^{\gamma}}{x_{4}^{\gamma}+E C_{50}^{\gamma}}\right),
$$

$B I S_{0}$ is the $B I S$ value of an awake patient typically set to $100, E C_{50}$ corresponds to drug concentration associated with $50 \%$ of the maximum effect and $\gamma$ is a parameter

\footnotetext{
${ }^{1}$ Tthe time dependence is omitted for simplicity of the notation.
} 
modeling the degree of non-linearity. Typical values for these parameters are $E C_{50}=$ $3.4 \mu \mathrm{g} / \mathrm{ml}$ and $\gamma=3$. Let us stress that the chosen three-compartment model (1) is one of the possible compartment models. Its simplicity and its good representativity have motived our choice even if there exist other models of different complexity for the Propofol - BIS relationship [13].

Finally, it is important to note that the state and the input of system (1) have to be positive, that is to respect the following constraints:

$$
\begin{aligned}
& x_{a n} \geq 0 \\
& u_{a n} \geq 0
\end{aligned}
$$

It is then important to observe that the system (1) and (4) enters in the class of positive systems. Furthermore, note that matrix $A_{0}$ is a Metzler matrix [3].

\subsection{Problem formulation}

One important fact regarding model (1) resides in the difference of dynamics: indeed, the dynamics of metabolism and circulation of Propofol in the central compartment and the site effect is ten times faster than in muscles, and even a hundred times faster than in fat. A classical way to address this kind of problem is to describe the system as a singularly perturbed system [12]. Hence, based on a singularly description [14], the blood and the effect site parts are gathered in the fast subsystem and the muscle and the fat parts in the slow subsystem. Then, system (1) can be rewritten as follows:

$$
\left[\begin{array}{c}
\dot{\bar{x}} \\
\varepsilon \dot{\bar{z}}
\end{array}\right]=\left[\begin{array}{ll}
A_{11} & A_{12} \\
A_{21} & A_{22}
\end{array}\right]\left[\begin{array}{l}
\bar{x} \\
\bar{z}
\end{array}\right]+\left[\begin{array}{l}
B_{1} \\
B_{2}
\end{array}\right] u_{a n}
$$

with

$$
\begin{aligned}
& A_{11}=\left[\begin{array}{cc}
-a_{21} & 0 \\
0 & -a_{31}
\end{array}\right] ; A_{12}=\left[\begin{array}{ll}
a_{12} & 0 \\
a_{13} & 0
\end{array}\right], B_{1}=\left[\begin{array}{l}
0 \\
0
\end{array}\right] \\
& A_{21}=\left[\begin{array}{cc}
\varepsilon a_{21} & \varepsilon a_{31} \\
0 & 0
\end{array}\right]=\varepsilon A_{21}^{0} ; A_{22}=\left[\begin{array}{cc}
-\varepsilon\left(a_{10}+a_{12}+a_{13}\right) & 0 \\
\varepsilon a_{e 0} / V_{1} & -\varepsilon a_{e 0}
\end{array}\right]=\varepsilon A_{22}^{0} \\
& B_{2}=\left[\begin{array}{l}
\varepsilon \\
0
\end{array}\right]=\varepsilon B_{2}^{0}
\end{aligned}
$$

where $\varepsilon>0, \bar{x}$ corresponds to the slow state and $\bar{z}$ corresponds to the fast state. $\varepsilon$ takes small values and corresponds to the perturbation parameter. Furthermore, the $B I S$ is rewritten in this case as:

$$
B I S\left(\bar{z}_{2}\right)=B I S_{0}\left(1-\frac{\bar{z}_{2}^{\gamma}}{z_{2}^{\gamma}+E C_{50}^{\gamma}}\right)
$$

where $\bar{z}_{i}, i=1,2$ are the components of vector $\bar{z}$. 
The following assumption holds.

Assumption 1 Matrix $A_{22}^{0}$ is non singular. Matrix $A_{22}$ is non singular for any $\varepsilon>0$.

In most of studies addressing the control design for singularly perturbed systems, the goal is to control the slow dynamics as the crucial problem [12]. In the case of the depth of anesthesia, the most important objective is the control of the fast dynamics because the regulation of the $B I S$ is a direct function of the concentration at the effect site and thus of the fast dynamics on which the administered drug directly acts.

Moreover, during a surgery, the $B I S$ must be brought then maintained close to 50 , or at least in an interval between 40 and 60. Due to relation (7) describing the relation between the $B I S$ and the effect site concentration, it follows that for the $B I S$ equal to $50 \%$ of $B I S_{0}$ the effect site concentration must be equal to $E C_{50}$. Then, the computation of the associated equilibrium point $\bar{x}_{e}, \bar{z}_{e}$ satisfying $\dot{\bar{x}}_{e}=0$ and $\dot{\bar{z}}_{e}=0$ gives:

$$
\begin{aligned}
& \bar{z}_{e 1}=V_{1} \bar{z}_{e 2} \\
& \bar{z}_{e 2}=E C_{50}\left(\frac{B I S_{0}}{B I S_{e}}-1\right)^{1 / \gamma} \\
& \bar{x}_{e 1}=\frac{a_{12}}{a_{21}} \bar{z}_{e 1} \\
& \bar{x}_{e 2}=\frac{a_{13}}{a_{31}} \bar{z}_{e 1} \\
& \bar{u}_{e}=a_{10} \bar{z}_{e 1}
\end{aligned}
$$

where $B I S_{e}$ denotes the desired value of the $B I S$ at the equilibrium and $\bar{x}_{i}, i=1,2$ are the components of vector $\bar{x}$.

Hence, we can define the error model around the equilibrium with $x=\bar{x}-\bar{x}_{e}$, $z=\bar{z}-\bar{z}_{e}$ and $u=u_{a n}-\bar{u}_{e}$ :

$$
\left[\begin{array}{c}
\dot{x} \\
\varepsilon \dot{z}
\end{array}\right]=\left[\begin{array}{ll}
A_{11} & A_{12} \\
A_{21} & A_{22}
\end{array}\right]\left[\begin{array}{l}
x \\
z
\end{array}\right]+\left[\begin{array}{l}
B_{1} \\
B_{2}
\end{array}\right] u
$$

with matrices defined in (6).

The problem we intend to solve in the paper can be summarized as follows.

Problem 1. Find a structured control gain $K$ :

$$
K=\left[0 K_{f}\right], K_{f} \in \mathbb{R}^{2 \times 2}
$$

such that:

1. The system (9)-(6) controlled through the control law $u=K_{f} z$ is asymptotically stable;

2. The positivity of $x_{a n}$ and $u_{a n}$ is ensured, or equivalently due to the change of variables around the equilibrium point $\left[\begin{array}{l}x \\ z\end{array}\right] \geq-\left[\begin{array}{l}\bar{x}_{e} \\ \bar{z}_{e}\end{array}\right]$ and $u \geq-\bar{u}_{e}$.

Note that to address Problem 1, the state of the fast subsystem is assumed to be available. 


\section{Main conditions}

In order to solve Problem 1, we consider in the sequel a procedure in two main steps: 1) we design the structured control gain to ensure the closed-loop asymptotic stability; and 2) we provide an analysis of the solution to ensure the constraints satisfaction.

\subsection{Theoretical conditions}

Let us introduce the following notation:

$$
\begin{aligned}
& A_{s}=A_{11}-A_{12} A_{22}^{-1} A_{21}=A_{11}-A_{12}\left(A_{22}^{0}\right)^{-1} A_{21}^{0} \\
& B_{s}=B_{1}-A_{12} A_{22}^{-1} B_{2}=B_{1}-A_{12}\left(A_{22}^{0}\right)^{-1} B_{2}^{0}
\end{aligned}
$$

From (9), the slow subsystem can be derived by considering $\varepsilon=0$ and expressing $z$ as a function of $x$ and $u$, which are denoted by $x_{s}$ and $u_{s}$, that is from Assumption 1:

$$
z_{s}=-A_{22}^{-1}\left(A_{21} x_{s}+B_{2} u_{s}\right)=-\left(A_{22}^{0}\right)^{-1}\left(A_{21}^{0} x_{s}+B_{2}^{0} u_{s}\right)
$$

In (12), $z_{s}$ can be interpreted as the slow part of $z$. By replacing $z_{s}$ in the original system, the slow dynamics reads:

$$
\dot{x}_{s}=A_{s} x_{s}+B_{s} u_{s}
$$

with $A_{s}$ and $B_{s}$ defined in (11). Similarly to define the fast dynamics, the vector $x$ is considered as constant (that is $x=x_{s}$ and $\dot{z}_{s}=0$ ) and we denote by $z_{f}=z-z_{s}$ and $u_{f}=u-u_{s}$ the fast part of the state and the control, respectively. Then, the fast dynamics reads:

$$
\dot{z}_{f}=A_{22}^{0} x_{f}+B_{2}^{0} u_{f}
$$

If the slow control $u_{s}$ and the fast one $u_{f}$ are determined, the complete control law is given by $u=u_{s}+u_{f}$.

Then by using a Lyapunov-based approach and adapting the results of [8] and [14], we can state the following conditions to solve item 1 of Problem 1.

Theorem 1. If there exist two symmetric positive definite matrices $W_{s} \in \mathbb{R}^{2 \times 2}, W_{f} \in$ $\mathbb{R}^{2 \times 2}$ and a matrix $S_{f} \in \mathbb{R}^{1 \times 2}$ satisfying the following inequalities:

$$
\begin{gathered}
\operatorname{He}\left(A_{22}^{0} W_{f}+B_{2}^{0} S_{f}\right)<0 \\
H e\left(A_{s} W_{s}-B_{s}\left(I+S_{f} W_{f}^{-1}\left(A_{22}^{0}\right)^{-1} B_{2}^{0}\right)^{-1} S_{f} W_{f}^{-1}\left(A_{22}^{0}\right)^{-1} A_{21}^{0} W_{s}\right)<0
\end{gathered}
$$

then the control gain as defined in (10) with $K_{f}=S_{f} W_{f}^{-1}$ solves item 1 of Problem 1 . 
Proof. This result is based on the use of Theorem 4 in [8] adapted to our case. Thus, we want to find a symmetric positive definite matrix $W_{0} \in \mathbb{R}^{4 \times 4}$ and a matrix $S_{0} \in \mathbb{R}^{1 \times 4}$ such that

$$
A_{\mathcal{\varepsilon}} W_{0}+W_{0} A_{\mathcal{\varepsilon}}^{T}+B_{\varepsilon} S_{0}+S_{0}^{T} B_{\varepsilon}^{T}<0
$$

where from (6)

$$
A_{\varepsilon}=\left[\begin{array}{cc}
A_{11} & A_{12} \\
\frac{A_{21}}{\varepsilon} & \frac{A_{22}}{\varepsilon}
\end{array}\right]=\left[\begin{array}{ll}
A_{11} & A_{12} \\
A_{21}^{0} & A_{22}^{0}
\end{array}\right] ; B_{\varepsilon}=\left[\begin{array}{c}
B_{1} \\
\frac{B_{2}}{\varepsilon}
\end{array}\right]=\left[\begin{array}{c}
B_{1} \\
B_{2}^{0}
\end{array}\right]
$$

By developing each terms of the matrix at the right-hand side of relation (17) and by using arguments as in [8], matrices $W_{0}$ and $S_{0}$ can be described as follows:

$$
\begin{aligned}
W_{0} & =\left[\begin{array}{cc}
W_{s} & -\left(A_{21}^{0} W_{s}+B_{2}^{0} S_{s}\right)^{T}\left(A_{22}^{0}\right)^{-T} \\
\star & W_{f}+\left(A_{22}^{0}\right)^{-1}\left(A_{21}^{0} W_{s}+B_{2}^{0} S_{s}\right) W_{s}^{-1}\left(A_{21}^{0} W_{s}+B_{2}^{0} S_{s}\right)^{T}\left(A_{22}^{0}\right)^{-T}
\end{array}\right] \\
S_{0} & =\left[\begin{array}{ll}
S_{s} & S_{f}-S_{s} W_{s}^{-1}\left(A_{21}^{0} W_{s}+B_{2}^{0} S_{s}\right)^{T}\left(A_{22}^{0}\right)^{-T}
\end{array}\right]
\end{aligned}
$$

where $W_{f}, S_{f}$ are solutions to relation (15) and $W_{s}, S_{s}$ solutions to

$$
\mathrm{He}\left(A_{s} W_{s}+B_{s} S_{s}\right)<0
$$

That corresponds to characterize a gain $K=S_{0} W_{0}^{-1}$ such that $A_{\varepsilon}+B_{\varepsilon} K$ is Hurwitz. From (19), one gets the following expression of $K$ :

$$
\begin{aligned}
& K=\left[\begin{array}{ll}
S_{s} S_{f}
\end{array}\right]\left[\begin{array}{cc}
W_{s}^{-1} & 0 \\
W_{f}^{-1}\left(A_{22}^{0}\right)^{-1}\left(A_{21}^{0} W_{s}+B_{2}^{0} S_{s}\right) W_{s}^{-1} W_{f}^{-1}
\end{array}\right] \\
& =\left[S_{s} W_{s}^{-1}+S_{f} W_{f}^{-1}\left(A_{22}^{0}\right)^{-1}\left(A_{21}^{0} W_{s}+B_{2}^{0} S_{s}\right) W_{s}^{-1} S_{f} W_{f}^{-1}\right]
\end{aligned}
$$

In order to obtain a gain $K$ structured as in (10), one has to satisfy:

$$
S_{s} W_{s}^{-1}+S_{f} W_{f}^{-1}\left(A_{22}^{0}\right)^{-1}\left(A_{21}^{0} W_{s}+B_{2}^{0} S_{s}\right) W_{s}^{-1}=0
$$

or equivalently

$$
S_{s}+S_{f} W_{f}^{-1}\left(A_{22}^{0}\right)^{-1}\left(A_{21}^{0} W_{s}+B_{2}^{0} S_{s}\right)=0
$$

which corresponds to

$$
\left(I+S_{f} W_{f}^{-1}\left(A_{22}^{0}\right)^{-1} B_{2}^{0}\right) S_{s}+S_{f} W_{f}^{-1}\left(A_{22}^{0}\right)^{-1} A_{21}^{0} W_{s}=0
$$

By denoting $K_{f}=S_{f} W_{f}^{-1}$, one can remark that relation (15) is equivalent to verify

$$
\operatorname{He}\left(\left(A_{22}^{0}+B_{2}^{0} K_{f}\right) W_{f}\right)<0
$$

that is matrix $\left(A_{22}^{0}+B_{2}^{0} K_{f}\right)$ is Hurwitz and therefore non singular. Then, one can observe that matrix $\left(I+S_{f} W_{f}^{-1}\left(A_{22}^{0}\right)^{-1} B_{2}^{0}\right)$ is also non singular by using the inverse matrix definition of $\left(A_{22}^{0}+B_{2}^{0} K_{f}\right)^{-1}$. Hence, relation (22) reads 


$$
S_{s}=-\left(I+S_{f} W_{f}^{-1}\left(A_{22}^{0}\right)^{-1} B_{2}^{0}\right)^{-1} S_{f} W_{f}^{-1}\left(A_{22}^{0}\right)^{-1} A_{21}^{0} W_{s}
$$

From (23), if relation (16) holds then relation (20) is verified.

As mentioned before, we need at this stage to ensure the satisfaction of item 2 of Problem 1. Actually, considering the controller issued from Theorem 1, we have to provide a stability analysis of the original system (1)-(2) by considering that the input can saturate as follows: $\left.u_{a n}=\operatorname{sat}\left(K x_{a n}\right)\right)$. Rather than addressing the problem in a linear framework (saturation not allowed), it is preferable to consider the problem in the saturated allowed framework. Depending on the controller designed the global asymptotic stability (GAS) or the local asymptotic stability (LAS) of the closed-loop system is achevied [18]. This is detailed in the following section.

\subsection{Computational issues}

The main drawback of Theorem 1 resides in the fact that relation (16) is nonlinear in the decision variables due to the presence of products between some variables, relation (15) being linear. Hence, the lack of linearity of this condition makes it not computationally tractable to obtain a solution to Problem 1 [4]. However, some relaxation steps can be proposed. Note that the first inequality (15) is linear in the decision variables $W_{f}, S_{f}$. The second inequality (16) is nonlinear in the decision variables $W_{s}, W_{f}, S_{f}$ but becomes linear in $W_{s}$ if $W_{f}$ and $S_{f}$ are fixed. Hence, one can consider the following first algorithm regarding the controller design procedure.

\section{Algorithm 1}

1. Select a desired decay rate for the fast subsystem with parameter $\mu_{f}>0$.

2. Compute $K_{f}=S_{f} W_{f}^{-1}$ stabilizing and improving the decay rate of the fast subsystem by solving

$$
H e\left(A_{22}^{0} W_{f}+B_{2}^{0} S_{f}+\mu_{f} W_{f}\right)<0
$$

3. Feasibility problem. Find $W_{s}$ solution to

$$
H e\left(A_{s} W_{s}-B_{s}\left(I+K_{f}\left(A_{22}^{0}\right)^{-1} B_{2}^{0}\right)^{-1} K_{f}\left(A_{22}^{0}\right)^{-1} A_{21}^{0} W_{s}\right)<0
$$

4. If (25) is feasible, then $K=\left[\begin{array}{ll}0 & K_{f}\end{array}\right]$ stabilizes the closed-loop system by acting on fast dynamics.

If not, then decrease the decay rate parameter $\mu_{f}$ and go back to step 2.

Remark 1. System (1) being open-loop stable, there always exists a solution to the feasibility linear problem (24)-(25) with $K_{f}=0$. Then, there always exists a $\mu_{f}$ small enough such that, for a controller issued from step 2, the LMI condition in step 3 is feasible.

From Algorithm 1, we have in hand the stabilizing controller, and we can now manage the constraints. A first direction could be to adapt the conditions provided in 
[14] to our current problem. Due to the difficulties encountered to deal with the nonlinearities appearing in the conditions, we decided here to propose an alternative route by providing analysis conditions based on tools issued from [18] and [19], by using the toolbox SATAW-Tool ${ }^{2}$.

\section{Algorithm 2}

1. Given the value of $K_{f}$ (and therefore of $K$ ) resulting from Algorithm 1.

2. Global asymptotically stability (GAS) case. Find a symmetric positive definite matrix $W \in \mathbb{R}^{4 \times 4}$ and a diagonal positive definite matrix $S \in \mathbb{R}^{1 \times 1}$ solution to the feasibility problem:

$$
\left[\begin{array}{cc}
W\left(A_{\varepsilon}+B_{\varepsilon} K\right)^{T}+\left(A_{\varepsilon}+B_{\varepsilon} K\right) W & B_{\varepsilon} S-W K^{T} \\
S B_{\varepsilon}^{T}-K W & -2 S
\end{array}\right]<0
$$

3. Local asymptotic stability (LAS) case. If the global case is unfeasible, given $u_{0}=\bar{u}_{e}$, find a symmetric positive definite matrix $W \in \mathbb{R}^{4 \times 4}$, a diagonal positive definite matrix $S \in \mathbb{R}^{1 \times 1}$, a matrix $Z \in \mathbb{R}^{1 \times 4}$ and a positive scalar $\gamma$ solution to the optimization problem:

$$
\begin{gathered}
\min -\operatorname{trace}(W)+\gamma \\
\text { s.t. } \\
{\left[\begin{array}{cc}
W\left(A_{\varepsilon}+B_{\varepsilon} K\right)^{T}+\left(A_{\varepsilon}+B_{\varepsilon} K\right) W & B_{\varepsilon} S-W K^{T}-Z^{T} \\
S B_{\varepsilon}^{T}-K W-Z & -2 S
\end{array}\right]<0} \\
{\left[\begin{array}{ll}
W & Z^{T} \\
Z & \gamma u_{0}^{2}
\end{array}\right] \geq 0}
\end{gathered}
$$

The objective of the optimization criterion considered in step 3 of Algorithm 2 is to maximize the region

$$
\mathscr{E}(W, \gamma)=\left\{\left[\begin{array}{l}
x \\
z
\end{array}\right] \in \mathbb{R}^{4} ;\left[\begin{array}{l}
x \\
z
\end{array}\right]^{T} W^{-1}\left[\begin{array}{l}
x \\
z
\end{array}\right] \leq \gamma^{-1}\right\}
$$

which is a region of invariance and asymptotic stability for the closed-loop system.

Remark 2 . The global condition does not depend on any bound $u_{0}$ and formally allows that non-symmetric bounds may be applied in practice. It also means that any initial condition may be applied, and, typically, formally guarantees that the controller may be applied from the patient awake state. On the other hand, the local condition is directly related to the bound $u_{0}=\bar{u}_{e}$, which means that, formally, $0 \leq u_{a n} \leq 2 \bar{u}_{e}$. Moreover, only initial states belonging to the set $\mathscr{E}(W, \gamma)$ should be considered.

Remark 3. One could also be interested in guaranteeing that, once the BIS enters the interval $[40,60]$, it remains inside this interval. Such a constraint could be added in the problems (26) and (27) through the additional condition:

\footnotetext{
${ }^{2}$ http ://homepages.laas.fr/queinnec/satawtool. html
} 


$$
E W E^{\prime} \leq \gamma z_{2 M}^{2}
$$

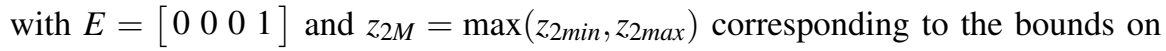
the effect site concentration $-z_{2 \min } \leq z_{2} \leq z_{2 \max }$ issued from the change of variables around the equilibrium point. However, this would result in drastically reducing the size of the region of invariance and asymptotic stability for the closed-loop system and would prevent to consider the patient awake state as initial state.

\section{Simulations}

To illustrate the approach let us consider a patient with the following characteristics: woman, 49 years old, $68 \mathrm{~kg}$ and $172 \mathrm{~cm}$. It corresponds to the system matrices defined with:

$$
\left[\begin{array}{l|l}
A_{11} & A_{12} \\
\hline A_{21}^{o} & A_{22}^{o}
\end{array}\right]=\left[\begin{array}{cc|cc}
-0.068 & 0 & 0.138 & 0 \\
0 & -0.004 & 0.077 & 0 \\
\hline 0.068 & 0.003 & -0.389 & 0 \\
0 & 0 & 0.042 & -0.456
\end{array}\right], \quad\left[\begin{array}{c}
B_{1} \\
\hline B_{2}^{o}
\end{array}\right]=\left[\begin{array}{c}
0 \\
0 \\
1 \\
0
\end{array}\right]
$$

For a target $B I S$ of 50 , the equilibrium point and associated input are given by

$$
\bar{x}_{e}=[69.5776809 .2000], \bar{z}_{e}=[36.76083 .4000], \bar{u}_{e}=6.7519
$$

and the open-loop spectrum of system (1) is equal to

$$
\lambda_{b o}=\{-0.002,-0.043,-0.415,-0.456\}
$$

First, Algorithm 1 allows to provide solution to the first objective of Problem 1. Considering various pole-placement constraints, the problem is feasible and one obtains:

$$
\begin{aligned}
& \mathrm{K} 1: \quad \mu_{f}=1.2 K_{f}=\left[\begin{array}{ll}
-3.3124 & -73.3247
\end{array}\right] \\
& \lambda_{b f}=\{-0.003,-0.067,-2.079 \pm 0.672 i\} \\
& \mathrm{K} 2: \quad \mu_{f}=1.3 K_{f}=\left[\begin{array}{ll}
-3.9172 & -102.4763
\end{array}\right] \\
& \lambda_{b f}=\{-0.003,-0.067,-2.382 \pm 0.780 i\} \\
& \mathrm{K} 3: \quad \mu_{f}=1.5 K_{f}=[-3.6796-118.7038] \\
& \lambda_{b f}=\{-0.003,-0.067,-2.263 \pm 1.317 i\} \\
& \mathrm{K} 4: \quad \mu_{f}=1.7 K_{f}=[-4.3694-167.6553] \\
& \lambda_{b f}=\{-0.003,-0.067,-2.608 \pm 1.560 i\}
\end{aligned}
$$

where $\lambda_{b f}$ denotes the closed-loop spectrum. Then Algorithm 2 is used to check if the closed-loop saturated system is globally asymptotically stable or, if not, if the 
system may be initialized from the patient awake state. It results that the saturated closed-loop system is GAS with controller K1, but only LAS with controllers K2, $\mathrm{K} 3$ and K4. With the controller $\mathrm{K} 2,-x_{\text {ane }}$ belongs to the associated set $\mathscr{E}(W, \gamma)$ and the controller may be safely applied with the patient initially awake. On the other hand, with the controllers $\mathrm{K} 3$ and $\mathrm{K} 4,-\bar{x}_{e}$ belongs to the associated set $\mathscr{E}(W, \gamma)$ but only a percentage of $-\bar{z}_{e}$ belongs to the set (40\% with $\mathrm{K} 3$, and $7 \%$ with $\left.\mathrm{K} 4\right)$.

Numerical simulations are plotted in Figure 4. In Figure 4(a), one can see the evolution of $B I S$ from the patient awake state to the reference 50, in open-loop (green) and in closed-loop with controllers K2 (blue), K3 (red) and K4 (black). The overshoot with controller $\mathrm{K} 4$ in not desirable as the $B I S$ goes temporarily below the bound 40. Figure 4(b) presents the associated input.

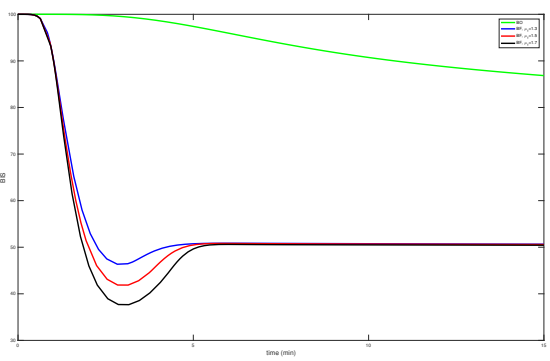

(a)

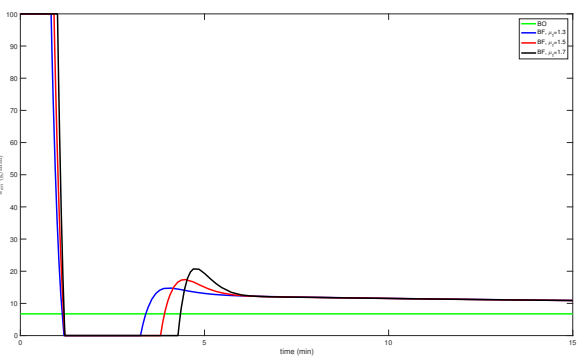

(b)

Fig. 1 Time simulation of the open-loop and saturated closed-loop systems with controllers K2, $\mathrm{K} 3$ and $\mathrm{K} 4$

\section{Conclusion}

Taking benefit from the singularly perturbed systems framework, the fast and slow dynamics present in the compartmental system have been separated. With the aim at accelerating the fast dynamics, the design of a structured state feedback controller has been first proposed. Second, some relaxation schemes associated to convex optimization problems allowed to guarantee the satisfaction of the constraints.

This work lets some questions open. In particular, one would be interested with more complete conditions not only to deal with the fast dynamics but also to guarantee that the constraints are satisfied and to initialize the system to the patient awake conditions, in order to mathematically validate the medical strategy from induction to maintenance. This will be the subject of future works. 


\section{References}

1. J.M. Bailey and W.M. Haddad. Drug dosing control in clinical pharmacology. IEEE Control Systems Magazine, 25(2):35-51, April 2005.

2. C. L. Beck. Modeling and control of pharmacodynamics. European Journal of Control, 24:33-49, 2015.

3. A. Berman, M. Neumann, and R.J. Stern. Nonnegative Matrices in Dynamic Systems. WileyInterscience, John Wiley and Sons, New York, USA, 1989.

4. S. Boyd, L. E. Ghaoui, E. Feron, and V. Balakrishnan. Linear Matrix Inequalities in System and Control Theory. Society for Industrial and Applied Mathematics, June 1997.

5. M. J. Coppens, D. J. Eleveld, J. H. Proost, L. A. Marks, J. F. Van Bocxlaer, H. Vereecke, A. R. Absalom, and M. M. Struys. An evaluation of using population pharmacokinetic models to estimate pharmacodynamic parameters for propofol and bispectral index in children. Anesthsiology, 115(1):83-93, 2011.

6. H. Derendorf and B. Meibohm. Modeling of pharmacokinetic / pharmacodynamic (pk/pd) relationships: Concepts and perspectives. Pharmaceutical Research, 16(2):176-185, 1999.

7. M. Fiacchini, I. Queinnec, S. Tarbouriech, and M. Mazerolles. Invariant based control of induction and maintenance phases for anesthesia. In 6th IFAC Conference on Foundations of Systems Biology in Engineering (FOSBE), MAgdeburg, Germany, October 2016.

8. G. Garcia and S. Tarbouriech. Control of singularly perturbed systems by bounded control. In American Control Conference, pages 4482-4487, Denver, USA, 2003.

9. A. Gentilini, C. Schaniel, M. Morari, C. Bieniok, R. Wymann, and T. Schnider. A new paradigm for the closed-loop intraoperative administration of analgesics in humans. IEEE Transactions on Biomedical Engineering, 49(4):289-299, 2002.

10. W. M. Haddad, V. Chellaboina, and Q. Hui. Nonnegative and Compartmental Dynamical Systems. Princeton, 2010.

11. W.M. Haddad, T. Hayakawa, and J. Bailey. Adaptive control for non-negative and compartmental dynamical systems with application on general anesthesia. International journal of adaptive control and signal processing, 17(3):209-235, March 2003.

12. P.V. Kokotovic, H.K Khalil, and J. O'Reilly. Singular Perturbation Methods in Control; Analysis and Design. New York: Academic, 1986.

13. J. M. Lemos, D. V. Caiado, B. A. Costa, L. A. Paz, T. F. Mendonca, S. Esteves, and M. Seabra. Robust control of maintenance-phase anesthesia. IEEE Control Systems Magazine, 34(6):2438, December 2014.

14. I. Lizarraga, S. Tarbouriech, and G. Garcia. Control of singularly perturbed systems under actuator saturation. In 16th World IFAC Congress, pages 243-248, Prague, Czech Republic, 2005.

15. C. F. Minto, T. W. Schneider, and S. L. Shafer. Pharmacokinetics and pharmacodynamics of remifentanil.Model application. Anesthesiology, 86(1):24-33, 1997.

16. T.W. Schnider, C.F. Minto, P.L. Gambus, C. Anderson, D.B. Goodale, and E. Young. The influence of method of administration and covariates on the pharmacokinetics of propofol in adult volunteers. The journal of american society of anesthesiologist, 88(5):1170-1182, May 1998.

17. K. Soltesz, J.-O. Hahn, G.A. Dumont, and J.M. Ansernimo. Individualized pid control of depth of anesthesia based on patient model identification during the induction phase of anesthesia. IEEE Conference on Decision and Control and European Control Conference (CDC-ECC) Orlando USA, 855-860, December 2011.

18. S. Tarbouriech, G. Garcia, J.M.. Gomes da Silva Jr., and I. Queinnec. Stability and Stalization of Linear Systems with Saturating Actuators. Springer, 2011.

19. S. Zabi, I. Queinnec, S. Tarbouriech, G. Garcia, and M. Mazerolles. New approach for the control of anesthesia based on dynamics decoupling. In 9th IFAC Symposium on Biological and Medical Systems (BMS 2015), Berlin, Germany, 2015.

20. Z.T. Zhusubaliyev, A. Medvedev, and M.M. Silva. Nonlinear dynamics in closed-loop anesthesia: Pharmacokinetic/pharmacodynamic model under pid-feedback. In American Control Conference, pages 5496-5501, Portland, USA, 2014. 